\title{
El coste del seguimiento farmacoterapéutico en una farmacia comunitaria (II): costes de funcionamiento del servicio
}

\author{
$M^{a}$ Belén Cobián Rodríguez \\ Doctora en Farmacia. Farmacéutica comunitaria en A Coruña.
}

\section{PALABRAS CLAVE}

seguimiento

farmacoterapéutico, recursos, coste, servicios en farmacia comunitaria

SFT: seguimiento farmacoterapéutico. PRM: problemas relacionados con los medicamentos.

RNM: resultados negativos de la medicación. SERGAS: Servizo Galego de Saúde.

\section{KEYWORDS}

pharmacotherapy follow-up, costs, resources, community pharmacy services

\section{RESUMEN}

Introducción: El seguimiento farmacoterapéutico es un servicio que atiende las necesidades de los enfermos crónicos polimedicados pero invierte muchos recursos en un solo paciente. El objetivo del estudio fue conocer las variaciones en la dispensación de medicamentos derivadas del seguimiento farmacoterapéutico y determinar los recursos invertidos en el funcionamiento y sus costes asociados.

Métodos: Se realizó el servicio de seguimiento farmacoterapéutico durante seis meses de una muestra de 20 pacientes seleccionados en función del riesgo de presentar RNM utilizando el método Dáder. Se registraron los recursos materiales, de información y humanos utilizados y se calculó su coste.

Resultados: El número de medicamentos dispensado a los pacientes del programa disminuyó un 8,4\%. El coste del servicio de SFT de un paciente medio fue de 87 euros en los seis meses de estudio, siendo mayor en los pacientes que presentaban un mayor riesgo de presentar RNM

Discusión: El servicio de SFT disminuye los ingresos de la farmacia al disminuir la dispensación de medicamentos y supone un coste para la farmacia relacionado fundamentalmente con el tiempo de trabajo de los farmacéuticos. Desde el punto de vista del pagador, el ahorro en medicamentos pagaría la mayor parte de este servicio.

The cost of pharmacotherapeutic follow-up in a community pharmacy (II): service operating costs

\section{ABSTRACT}

Introduction: Pharmacotherapy follow-up is a service that meets the needs of polymedicated patients but invests a lot of resources. The aim of this study was to determine the changes in drug dispensation due to pharmacotherapy follow-up and the resources invested in the service and its associated costs.

Methods: Twenty patients in risk of drug related problems entered a pharmacotherapy follow-up program for six months using Dader's method. Material, information and human resources were registered and their cost calculated.

Results: Number of dispensed drugs to the patients in the program decreased $8.4 \%$. Pharmacotherapy follow-up costs for each patient in the program was 87 euros and increased as did drug related problems risk factors.

Discussion: Pharmacotherapy follow-up decreases pharmacy incomes on decreasing drug dispensation. Besides, pharmacist's working time increases, as well as its costs. A pharmacotherapy follow-up service in this pharmacy could nearly be paid by the medicines saved.

El estudio ha sido realizado con datos recogidos en la elaboración de la tesis doctoral de la autora: Diseño, implantación y análisis de un modelo para la gestión del seguimiento farmacoterapéutico en una farmacia comunitaria. Los costes del seguimiento farmacoterapéutico han sido actualizados a mayo de 2013.

Parte de los datos expuestos se han utilizado en la ponencia Gestión del seguimiento farmacoterapéutico en una farmacia comunitaria: Formación de los profesionales. II Congreso Nacional de Farmacéuticos Comunitarios (SEFAC) celebrado en A Coruña 2006.

Financiación: Ninguna ajena.

Conflicto de intereses: La autora declara no existir ningún conflicto de intereses en relación con el contenido del presente artículo. Cite este artículo como: Cobián MB. El coste del seguimiento farmacoterapéutico en una farmacia comunitaria (II): costes de funcionamiento del servicio. Farmacéuticos Comunitarios. 2014 Sep 01; 6(3):25-30. doi:10.5672/FC.2173-9218.(2014/Vol6).003.04 


\section{Introducción}

Si hay algo que tiene que caracterizar a un ejercicio profesional es su continua evolución para adaptarse a las necesidades de la sociedad a la que sirve. Esto, en el ámbito de una farmacia comunitaria de barrio en Galicia, que atiende a una población envejecida, polimedicada y con un nivel socioeconómico medio-bajo, se traduce en comprobar que los medicamentos que utiliza son necesarios, efectivos y seguros, y que todas sus enfermedades están siendo tratadas de la mejor manera posible. Un medio para conseguirlo es el seguimiento farmacoterapéutico (SFT), que ha sido definido como "el servicio profesional que tiene como objetivo la detección de problemas relacionados con los medicamentos (PRM) para la prevención y resolución de resultados negativos asociados a la medicación (RNM). Este servicio implica un compromiso y debe de proveerse de forma continuada, sistematizada y documentada, en colaboración con el propio paciente y con los demás profesionales del sistema de salud, con el fin de alcanzar resultados concretos que mejoren la calidad de vida del paciente" (1). Se trata de un servicio que invierte muchos recursos en un solo paciente y por lo tanto tiene que ser cuidadosamente gestionado.

De la misma manera que es necesario utilizar un enfoque ordenado y sistemático para el tratamiento de un paciente, también es preciso disponer de un enfoque ordenado y sistemático para la organización de un servicio de SFT (2), que posibilite que todos los pacientes que lo necesiten -en función de criterios definidos- puedan tener acceso a él. En la planificación del servicio se decidió utilizar los factores de riesgo de presentar RNM, que ya se habían estudiado en la farmacia (3), para establecer los criterios de selección de pacientes. La necesidad de control de PRM de estos pacientes no estaba cubierta por ningún profesional sanitario en esos momentos y además se contaba con una metodología de trabajo, proporcionada por el método Dáder (4), y con un numeroso grupo de pacientes con las características necesarias para entrar en el programa.

Por último hay que señalar que el farmacéutico comunitario español percibe unos honorarios proceden- tes del margen sobre el precio de los medicamentos dispensados (5). Por lo tanto, con el fin de determinar el coste de funcionamiento de un servicio de SFT fue necesario conocer las variaciones en la dispensación de medicamentos derivadas de dicho servicio y determinar los recursos invertidos y sus costes correspondientes, que constituyen los objetivos de este trabajo.

\section{Material y métodos}

En la farmacia se realizó el SFT durante seis meses de una muestra de 20 pacientes seleccionados en función de los siguientes criterios de inclusión:

- Ser enfermos crónicos y usuarios habituales de la farmacia.

- Tener una ficha de registro de medicamentos de al menos un año.

- Presentar al menos tres de los criterios considerados factores de riesgo de resultados negativos de la medicación.

- No estar ni haber estado incluidos en el programa de SFT existente en la farmacia.

A los pacientes que acudieron a la farmacia y que, a juicio de las farmacéuticas, podrían cumplir estos criterios de inclusión, se les ofertó el servicio hasta que 20 de ellos realizaron la entrevista inicial de la que se dedujo que presentaban al menos tres de las siguientes condiciones los siguientes: Edad mayor de 65 años, Sexo femenino, Nivel socioeconómico bajo, Polimedicación (utilización de cinco o más medicamentos durante al menos seis meses), Número de prescriptores superior a dos, No Conocimiento de su medicación e Incumplimiento del tratamiento prescrito.

Se utilizó el método Dáder de SFT (4).

Con el fin de evaluar la variación en la dispensación de medicamentos derivada del SFT se cuantificó la medicación que estaban utilizando los pacientes al principio $\mathrm{y}$ al final del periodo de SFT.

Se registraron los recursos materiales, de información y humanos utilizados en el SFT de los pacientes en este periodo.

Los recursos humanos fueron valorados en función del tiempo necesario para realizar los distintos subprocesos del SFT de los pacientes.
Se calculó el coste de la medicación dispensada a los pacientes en los seis meses de SFT utilizando para ello el precio medio de la receta de pensionistas en la farmacia de estudio en los primeros cinco meses de 2013. Este fue de $14,85 €$.

Se estudió el coste asociado a la utilización de recursos materiales y de información.

Se calculó el coste de los recursos humanos, utilizando para ello el convenio vigente en 2013 y se consideró así mismo el coste de la seguridad social de las farmacéuticas adjuntas, lo que supuso un coste por hora trabajada de $18,53 €$.

En el análisis descriptivo se utilizaron porcentajes para variables cualitativas, y medidas centrales, media, con medidas de dispersión, desviación estándar, para variables cuantitativas.

\section{Resultados}

La variación en el número de medicamentos dispensados derivada del SFT osciló entre 4 y 14, con una media de 9,0 $\pm 2,9$ en el momento de la entrevista inicial y $8,2 \pm 2,4$ al finalizar el estudio. El número de medicamentos de cada paciente al comienzo y al final del periodo de seguimiento está representado en la figura 1. Supuso una disminución en el número de medicamentos dispensados a los pacientes en SFT de un 8,4\%. Utilizando el precio medio por receta de pensionista de la farmacia, $14,85 €$, eso supuso una disminución de la facturación de $11,10 €$ por paciente y mes.

Los recursos utilizados en el servicio de SFT y el coste de los mismos fueron los siguientes:

En lo referente a recursos materiales, la utilización de las dos Zonas de Atención Personalizada (ZAP) existentes en la farmacia no supuso ningún coste apreciable en relación con el necesario para el normal funcionamiento de la farmacia.

En cuanto a la utilización de los recursos de información está recogida en la tabla 1. La utilización de estas fuentes de información no supuso un coste apreciable que pudiera ser atribuido al servicio.

En cuanto a los recursos humanos, el tiempo de SFT total fue de 93 horas y 58 minutos, lo que presenta un coste asociado de $1.740 €$. Esto se traduce en un tiempo medio por paciente durante los seis meses de 4 horas y 42 minutos, 


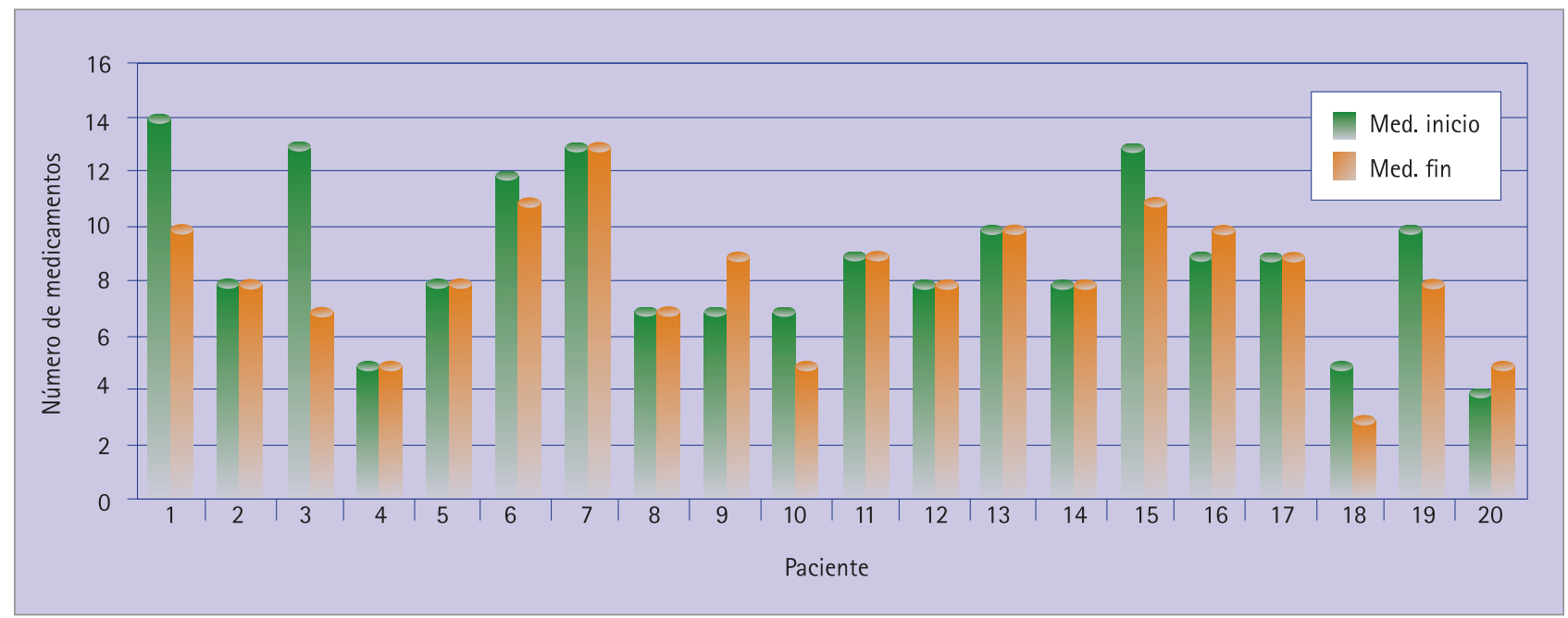

Figura 1 Número de medicamentos por paciente al principio y al final del periodo de estudio

Tabla 1 Utilización de los recursos de información

\begin{tabular}{|l|l|c|}
\hline \multicolumn{1}{|c|}{ Tipo de recurso de información } & Descripción del recurso & $\begin{array}{c}\text { \% de } \\
\text { pacientes } \\
\text { en los que } \\
\text { se utilizan }\end{array}$ \\
\hline Registros electrónicos & Ficha de utilización de medicamentos & $100 \%$ \\
\hline $\begin{array}{l}\text { Información de otros profesionales } \\
\text { sanitarios }\end{array}$ & Informes médicos y pruebas analíticas & $90 \%$ \\
\hline \multirow{2}{*}{ Información de medicamentos } & Entrevistas personales & $60 \%$ \\
\hline & Fuentes bibliográficas & $100 \%$ \\
\cline { 2 - 3 } & Centro de información de medicamentos & $30 \%$ \\
\hline
\end{tabular}

lo que supone un coste medio de $87,2 €$ por paciente.

El coste del proceso del servicio de SFT de cada paciente está reflejado en la figura 2.

Por otra parte, encontramos que están directamente relacionados el número de factores de riesgo que presentan los pacientes con el tiem- po medio invertido en su SFT y, por lo tanto, con el coste del mismo. Los resultados se presentan en la tabla 2. tiempo del SFT en cada una de las fases que se lleva a cabo al realizarlo:

- Tiempo de realización de las entrevistas iniciales. Se realizaron un total de 20 entrevistas iniciales con
En cuanto a la distribución del un tiempo medio de $30,5 \pm 19,1 \mathrm{mi}-$ nutos.

- Tiempo de elaboración de los estados de situación. Se elaboraron un total de 71 estados de situación, una media de $3,6 \pm 2,4$ por paciente. El tiempo medio necesario para elaborar un estado de situación fue de 10,6 minutos y el tiempo medio por paciente en los seis meses del programa fue de $37,7 \pm 27,7 \mathrm{minu}$ tos.

- Tiempo de realización de las evaluaciones globales. Se realizaron un total de 71 evaluaciones globales, el tiempo medio empleado en la realización de las mismas fue de 29 minutos por cada una. Esto supone una media de $103,2 \pm 62,8 \mathrm{mi}-$ nutos, es decir, 1 hora y 43 minutos por paciente.

- Tiempo de las intervenciones farmacéuticas. Se efectuaron un total

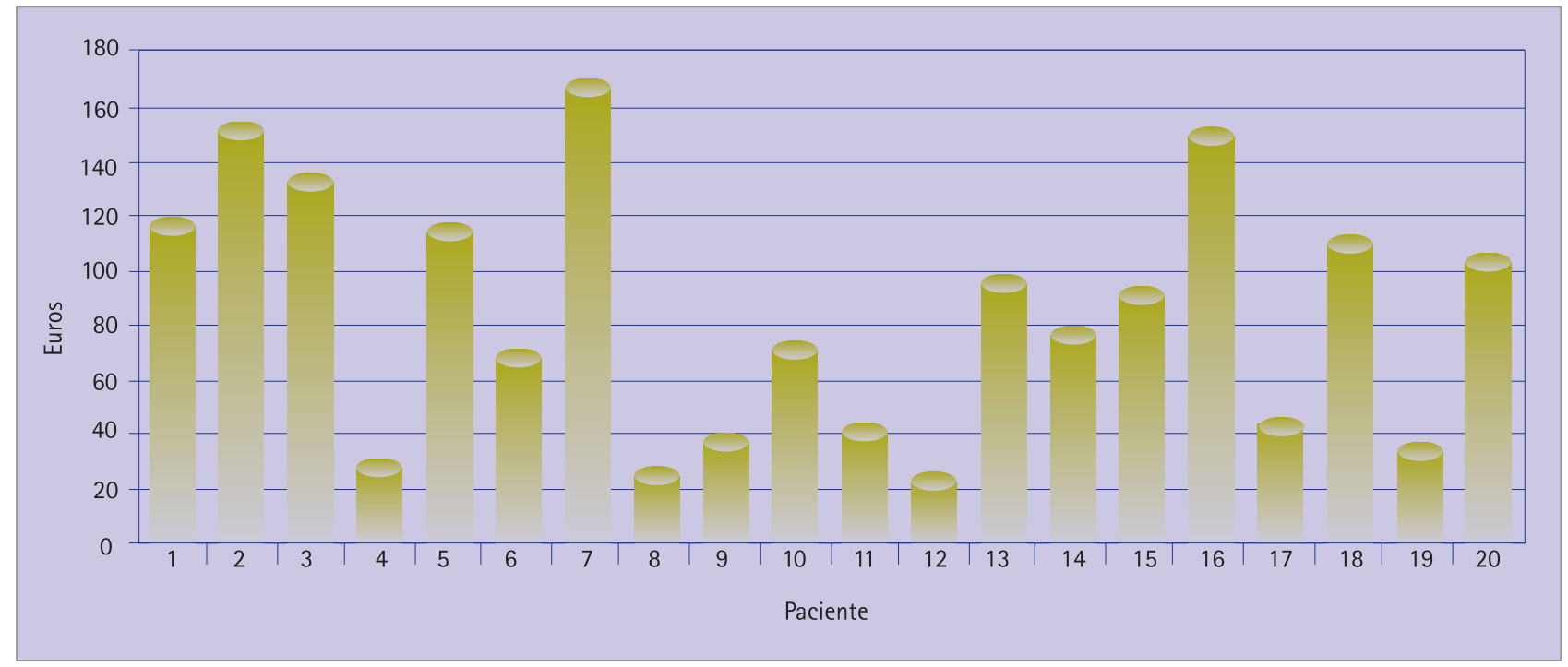

Figura 2 Coste del servicio de SFT en cada paciente en euros 
Tabla 2 Costes medios por paciente según factores de riesgo en los seis meses

\begin{tabular}{|c|c|c|}
\hline No factores riesgo & Tiempo/paciente & Coste/paciente \\
\hline 3 & 2 horas y 42 minutos & $50,0 €$ \\
\hline 4 & 4 horas y 46 minutos & $88,3 €$ \\
\hline 5 & 5 horas y 1 minutos & $93,0 €$ \\
\hline 6 & 6 horas y 40 minutos & $123,5 €$ \\
\hline
\end{tabular}

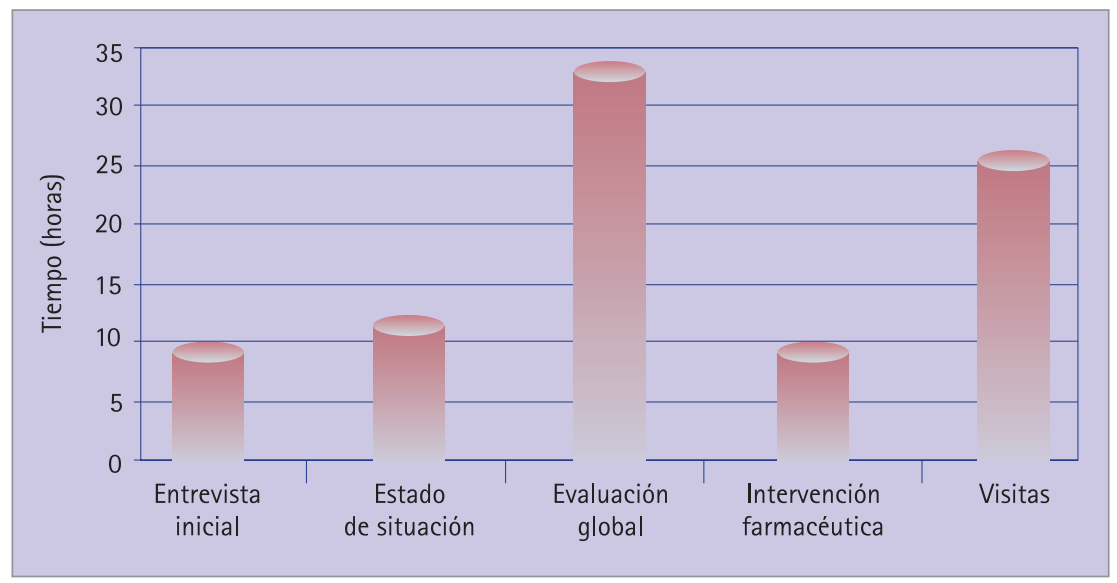

Figura 3 Distribución del tiempo de SFT en las distintas fases del servicio

de 47 intervenciones farmacéuticas, para resolver alguno de los RNM detectados, a lo largo de los seis meses que duró el programa. Esto supuso una media de 2,4 $\pm 2,5$ intervenciones por paciente. El tiempo medio de una intervención farmacéutica fue de 13,3 minutos y el tiempo medio por paciente correspondiente a ellas fue de 30,5 $\pm 38,6$ minutos.

- Tiempo de las visitas del seguimiento farmacoterapéutico. Se realizaron un total de 172 visitas, lo que supone una media de
$8,6 \pm 3,8$ visitas por paciente a lo largo de los seis meses del programa. El tiempo medio de cada visita fue de 9,3 $\pm 2,8$ minutos $y$ el tiempo medio por paciente, en los seis meses del programa, de $80,1 \pm 39,4$ minutos

El tiempo invertido en cada fase del servicio de SFT de los 20 pacientes está representado en la figura 3.

El tiempo y coste asociado al servicio de SFT y a cada una de sus fases se recogen en las tablas 3 y 4 .

Tabla 3 Tiempo y costes asociados al servicio de SFT

\begin{tabular}{|l|c|c|}
\hline & Tiempo & Coste \\
\hline SFT de 20 pacientes & 93 horas y 58 minutos & $1.740 €$ \\
\hline Media por paciente & 4 horas y 42 minutos & $87 €$ \\
\hline
\end{tabular}

Tabla 4 Coste de cada una de las fases del SFT

\begin{tabular}{|l|c|c|}
\hline \multicolumn{1}{|c|}{ Fase del proceso de SFT } & Coste medio por unidad & $\begin{array}{c}\text { Coste medio por paciente } \\
\text { en seis meses }\end{array}$ \\
\hline Entrevista inicial & $9,4 €$ & $9,4 €$ \\
\hline Estados de situación & $3,3 €$ & $11,6 €$ \\
\hline Evaluaciones globales & $9,0 €$ & $31,9 €$ \\
\hline Intervenciones farmacéuticas & $4,0 €$ & $9,4 €$ \\
\hline Visitas & $2,9 €$ & $24,7 €$ \\
\hline
\end{tabular}

\section{Discusión}

En los últimos años diversas administraciones han desarrollado programas de atención a enfermos crónicos pluripatologicos o polimedicados $(6,7)$. En la valoración de la medicación se utilizan criterios como Beers (8) o STOPP-START (9-11) que se aplican a los historiales farmacoterapéuticos de los pacientes diana. Otros abordajes posibles son métodos como Dáder (4) de SFT que basa su eficacia en la entrevista del profesional con el paciente y permite detectar PRM/RNM además de ayudar a solucionarlos.

En nuestro estudio, los pacientes fueron seleccionados en función de factores de riesgo de presentar PRM/ RNM, entre los que figuraban la polimedicación y el número de prescriptores mayor de dos, ambos factores estrechamente relacionados con la pluripatología. Estos criterios de selección hicieron que se atendiesen casos complejos, lo que hay que tener en cuenta a la hora de interpretar los resultados.

El número medio de medicamentos por paciente disminuyó entre el principio y el fin del periodo de estudio, pasando de una media de 9,0 medicamentos por paciente a 8,2 transcurridos los seis meses. Este dato concuerda con otros estudios realizados (12). Desde un punto de vista económico, además de un ahorro para el sistema sanitario, supone una disminución de ingresos para la farmacia, puesto que deja de ingresar el margen correspondiente a los medicamentos que no dispensa. Este hecho ha sido señalado por la Sociedad Española de Farmacia Comunitaria como una contradicción del sistema de retribución de la farmacia española y argumentado para su propuesta de sistema de retribución alternativo (13).

En el análisis de la utilización de recursos en el SFT de los pacientes hay que señalar que la utilización de recursos materiales no supuso un coste adicional para la farmacia ya que no se apreció aumento del consumo de electricidad, teléfono, fax, conexión a internet, etc. relacionado con el servicio profesional farmacéutico de SFT.

Los recursos de información fueron proporcionados por los propios pacientes, sus cuidadores, los demás profesionales sanitarios, la bibliografía existente en la farmacia y el 
Centro de Información de Medicamentos del Colegio de Farmacéuticos de A Coruña, y no supusieron coste alguno para la farmacia. La mayoría de los pacientes pudieron presentar información procedente de otros profesionales sanitarios, fundamentalmente informes de altas hospitalarias y de consultas a servicios de urgencia, lo que era previsible ya que se trataba de pacientes con múltiples factores de riesgo que habían tenido contacto reciente con los servicios de urgencias. La receta electrónica gallega (14) recoge la posibilidad de que el farmacéutico tenga un acceso limitado al historial farmacoterapéutico del paciente lo que resulta insuficiente para realizar un adecuado SFT. Para ello se hace necesario compartir información sobre síntomas y datos clínicos, resultados de pruebas diagnósticas, diagnósticos del paciente, tratamientos instaurados, dosificación y rangos de dosis posibles, inefectividad de la medicación, problemas de salud referidos por el paciente y no tratados, contraindicaciones, interacciones, incumplimiento de tratamientos y efectos adversos (15). Por otra parte, las experiencias de colaboración entre médicos y farmacéuticos para la atención al paciente, han producido resultados positivos (16-19) y la gestión de las enfermedades crónicas requiere de un trabajo conjunto del equipo de salud (20).

Los recursos humanos fueron los determinantes para realizar el SFT de los pacientes. Así, el tiempo efectivo dedicado a SFT fue de 93 horas y 58 minutos, lo que supuso una media de 4 horas y 42 minutos por paciente durante los seis meses en los que se registraron los recursos empleados en el servicio de SFT. Esto supuso un coste medio por paciente de $87 €$ en los seis meses. Sin embargo, dicho coste presentó importantes diferencias entre los pacientes, aumentando en relación al número de factores de riesgo de cada paciente, como era de esperar.

Existe una diferencia notable con el coste del servicio de SFT resultante del estudio conSIGUE (21), de 23,25€ paciente/mes, que habrá que analizar cuando se conozca el detalle de los indicadores utilizados.

En la relación pormenorizada de los tiempos dedicados a las distintas fases del seguimiento, las entrevistas iniciales consumieron un tiempo de
30,5 minutos por paciente. La previsión de media hora por paciente para realizar la entrevista inicial parece adecuada ya que coincide con el tiempo registrado en otros estudios de recursos utilizados en el SFT realizados a pacientes institucionalizados (22).

Se elaboraron, de media, 3,6 estados de situación por paciente, con sus correspondientes evaluaciones globales y ambas fases tuvieron un coste medio asociado de 11,60 y 31,90€ por paciente respectivamente. Este coste seguramente se reducirá al aumentar la experiencia de las farmacéuticas.

Las intervenciones farmacéuticas que se realizaron fueron, de media, 2,4 por paciente y costaron 9,40€. Nos parece imposible realizar la intervención farmacéutica medida en este estudio en 1,5 minutos, que es la referida en el estudio antes señalado (22), lo que sugiere que pese a utilizar el mismo método de SFT, los procedimientos aplicados no han sido los mismos. Además hay que señalar que en dicho estudio no se valora el tiempo invertido en realizar las visitas de SFT por lo que sospechamos que éstas no se realizan. En nuestro estudio, la supresión de las visitas disminuiría el coste en 24,7 euros por paciente en los seis meses.

En este sentido, existen distintos conceptos en los acuerdos sobre revisión de la medicación a los que se ha llegado en los diferentes países (23-25). En realidad, un proceso de SFT sin visitas consecutivas sería equivalente a lo que se conoce como revisión de la medicación en el consenso americano (25): un proceso sistemático de recogida de información especifica del paciente, evaluación de su medicación para identificar PRM, desarrollo de una lista priorizada de PRM y creación de un plan para resolverlos. También se correspondería con los niveles I o II de los servicios de revisión de medicación de British Columbia (24).

Con los datos de coste de cada una de las fases del proceso y para pacientes con el riesgo de RNM considerado como criterio de inclusión, el coste medio de un paciente, que incluya una entrevista inicial, un estado de situación, una evaluación global y una intervención farmacéutica sería de $25,7 €$.

El ahorro medio para el pagador (en nuestro caso el Servizo Ga- lego de Saúde SERGAS) de la medicación fue de $11,1 €$ por paciente y mes. El coste medio del servicio de seguimiento farmacoterapéutico durante 6 meses fue de $87 €$, por lo tanto, en esta farmacia y para este tipo de pacientes, el coste de este servicio profesional farmacéutico que supuso una disminución de ingresos, en caso de ser retribuido por el pagador, podría ser financiado en su mayor parte por la disminución de utilización de medicamentos.

\section{Referencias bibliográficas}

1. Foro de Atención Farmacéutica. Documento de Consenso. Madrid: Ed. Consejo General de Colegios Oficiales de Farmacéuticos. Madrid; 2008.

2. Cipolle RJ, Strand LM, Morley PC. Pharmaceutical care practice. New York; MacGrow-Hill: 1998.

3. Cobián B. Factores de Riesgo asociados a Problemas Relacionados con los Medicamentos. Farmacia Cobián, A Coruña. [Trabajo de investigación tutelado]. Santiago de Compostela: Universidad de Santiago de Compostela; 2001.

4. Sabater D, Silva MM, Faus MJ. Método Dáder. Guía de Seguimiento Farmacoterapéutico. Tercera edición. Granada: Grupo de Investigación en Atención Farmacéutica (CTS-131). Universidad de Granada; 2007.

5. Real Decreto 823/2008, de 16 de mayo, por el que se establecen los márgenes, deducciones y descuentos correspondientes a la distribución y dispensación de medicamentos de uso humano. BOE núm. 131, de 30 de mayo de 2008, páginas 25138 a 25140.

6. Ministerio de Sanidad, Servicios Sociales e Igualdad. Estrategia para el abordaje de la cronicidad en el Sistema Nacional de Salud [Internet]. Madrid; 2012. [Citado 17 Jun 2013]. Disponible en: http://www.msps.es/ organizacion/sns/planCalidadSNS/ pdf/ESTRATEGIA_ABORDAJE_CRONICIDAD.pdf

7. Mosquera R, Domínguez N, Lado E, Monteagudo J. La visión en Galicia sobre la asistencia al paciente crónico y pluripatológico; del hospital al sistema sanitario del futuro: Estrategia SERGAS 2014. Galicia Clin 2012;73(Supl.1):S42-S47.

8. American Geriatrics Society 2012 Beers Criteria Update Expert Panel. J Am Geriatr Soc. 2012;60(4):616-631.

9. Barry PJ, Gallagher P, Ryan C, O'Mahony D. START (Screening Tool to Alert doctors to the Right Treatment) 
- an evidence-based screening tool to detect prescribing omissions in elderly patients. Age Ageing. 2007;36: 632-638. doi:10.1093/ageing/afm 118

10 Gallagher P, 0'Mahony D. STOPP (Screening Tool of Older Persons' potentially inappropriate Prescriptions): application to acutely ill elderly patients and comparison with Beers' criteria. Age Ageing. 2008;37:673-679. doi:10.1093/ageing/afn 197

11. Gallagher P, Ryan C, Byrne S, Kennedy J, O'Mahony D. STOPP (Screening Tool of Older Person's Prescriptions) and START (Screening Tool to Alert doctors to Right Treatment). Consensus validation. Int $\mathrm{J}$ Clin Pharmacol Ther. 2008;46:72-83. doi:10.5414/ CPP46072

12. Benrimoj S, Gastelurrutia M, Farragher T, Feletto E, García P, Sáez-Benito L, et al. Resultados de la primera fase del programa conSIGUE: Estudio piloto: Medida del impacto clínico, económico y humanístico del servicio de seguimiento farmacoterapéutico en mayores polimedicados. Diciembre 2010. [Citado 17 Jun 2013]. Disponible en: http://www.portalfarma.com/ inicio/atencionfarmaceutica/Documents/consigue.pdf

13. Comisión de sistemas retributivos de Sefac. Propuesta de sistema retributivo de la Sociedad Española de Farmacia Comunitaria para la prestación del servicio de dispensación de medicamentos y productos sanitarios al Sistema Nacional de Salud. [citado 17 Jun 2012]. Disponible en: http://www.sefac.org/documentos/34
14. Decreto 206/2008, do 28 de agosto, de receita electrónica. DOG num. 181, de 18 de setembro de 2008; p.17252-6

15. Baena I, Martínez J, Faus MJ, Martínez F. Seguimiento farmacoterapéutico integral de pacientes en el sistema sanitario. Pharm Care Esp. 2002;4:325-332.

16. Álvarez de Toledo F, Arcos P, Eyaralar T, Abal F, Dago A, Cabiedes L, et al. Atención farmacéutica en personas que han sufrido episodios coronarios agudos (Estudio Tomcor). Rev Esp Salud Pública. 2001;75:375-87. doi:10.1590/S113557272001000400011

17. Sorensen L, Stokes JA, Purdie DM, Woodward M, Elliot R, Roberts MS. Medication reviews in the community: results of a randomized, controlled effectiveness trial. Br J Clin Pharmacol. 2005 Mar;59(3):376.

18. Doucette WR, McDonough RP, Klepser D, McCarthy R. Comprehensive medication therapy management: identifying and resolving drug-related issues in a community pharmacy. Clin Ther. 2005;27(7):1104-1111. doi:10.1016/ S0149-2918(05)00146-3

19. Isetts BJ, Schondelmeyer SW, Artz MB, Lenarz LA, Heaton AH, Wadd WB, et al. Clinical and economic outcomes of medication therapy management services: The Minnesota experience. J Am Pharm Assoc. 2008;48:203-214. doi:10.1331/JAPhA.2008.07108

20. Leonard M, Graham S, Bonacum D. The human factor: the critical importance of effective teamwork and communication in providing safe care. Qual Saf Health Care. 2004;13(1):i85-i90. doi:10.1136/qshc.2004.010033
21. Consejo General de Colegios Oficiales de Farmacéuticos [Internet]. Resumen y conclusiones de conSIGUE Impacto (2014). [Citado 18 Jun 2014]. Disponible en: http://www.portalfarma.com/ profesionales/investigacionfarmacia/ consigue/documents/14-03-27-resumen-consigue-impacto-divulgacion. pdf

22. Jódar F, García L, Martín JJ, López del Amo M, Karlsdotter K [Internet]. Evaluación económica del seguimiento farmacoterapéutico en los mayores institucionalizados en Andalucía. XXXV Reunión Anual Estudios Regionales. Valencia, 25 a 28 de noviembre de 2009. [Citado 22 Ene 2010]. Disponible en: www.reunionesdeestudiosregionales.org/valencia2009/htdocs/pdf/p113.pdf

23. Doucette WR, McDonough RP, Klepser D, McCarthy R. Comprehensive medication therapy management: identifying and resolving drug-related issues in a community pharmacy. Clinical Therapeutics 2005;27(7):1104-1111. doi:10.1016/S0149-2918(05)00146-3

24. British Columbia. Ministry of Health [Internet]. Medication review services. Policies, procedures and guidelines for pharmacists. V.2.2. 2012. [Citado 18 Jun 2013]. Disponible en: http://www.health.gov.bc.ca/pharmacare/pdf/medrevguide.pdf

25. Core Elements of an MTM Service Model Medication Therapy Management in Pharmacy Practice: Version 2.0.2008 [Internet]. [Citado 17 Jun 13]. Disponible en: http://www.accp. com/docs/positions/misc/CoreElements.pdf 